\title{
DISTRIBUIÇÃO ESPACIAL DAS DOENÇAS TROPICAIS NEGLIGENCIADAS NA REGIÃO OESTE DO ESTADO DE SÃO PAULO
}

\author{
Antonio Chigogoro Titosse \\ Mestre em Meio Ambiente e Desenvolvimento Regional - Universidade de Oeste Paulista (UNESP). \\ Biólogo - Universidade Pedagógica de Moçambique; Ministério da Saúde de Moçambique \\ amucovatitosse91@gmail.com \\ Elivelton da Silva Fonseca \\ Ph.D. em Geografia na Faculdade de Ciências e Tecnologia \\ Universidade Estadual Paulista (UNESP) \\ elivelton.fonseca@gmail.com
}

Marcus Vinicius Pimenta Rodrigues

Docente permanente do Programa de Pós-Graduação (mestrado e doutorado) em Meio Ambiente e Desenvolvimento Regional - MMADRE e docente colaborador do Mestrado em Ciências da Saúde da Universidade do Oeste Paulista (UNOESTE) marcusvinicius@unoeste.br

Ana Paula Marques Ramos Professora adjunta do Programa de Pós-graduação em Meio Ambiente e Desenvolvimento Regional Universidade do Oeste Paulista (UNOESTE) anapaula@unoeste.br

Rogério Giuffrida Professor do Curso de Medicina Veterinária e do Programa de Pos Gradução em meio Ambiente e Desenvolvimento Regional Universidade do Oeste Paulista (UNOESTE) rogeriogilfrida@unoeste.br

Alba Regina Azevedo Arana Coordenadora do Mestrado em Meio Ambiente e Desenvolvimento Regional. Diretora da Faculdade de Artes, Ciencia, Letras e Educação. Coordenadora do Curso de Geografia

Universidade do Oeste Paulista (UNOESTE) alba@unoeste.br

\begin{abstract}
RESUMO
Este artigo tem como objetivo caracterizar a incidência de doenças tropicais negligenciadas na região do oeste do estado de São Paulo entre 2007 e 2017. Trata-se de um estudo ecológico descritivo, com abordagem espaço-temporal, tendo por fonte de dados o Sistema de Agravos de Notificação (SINAN), o Instituto Brasileiro de Geografia e Estatística (IBGE). Foram elencadas 5 doenças: Dengue, Leishmaniose, Tuberculose, Hanseníase e Esquistossomose nos 45 municípios do Estado de São Paulo que compõe a Rede Regional de Assistência a Saúde (RRAS) de número 11, a RRAS11. A taxa de incidência das doenças elencadas em cada um dos 45 municípios da RRAS foi calculada através da razão entre o número de casos da doença e o número de habitantes no município em 2010. Através das taxas de incidência foi possível observar uma distribuição heterogênea dessas doenças nos 45 municípios em estudo, com maior incidência, em toda a região, de dengue e tuberculose. A comparação das taxas de incidência entre os diferentes municípios do Oeste Paulista nos permitiu identificar as populações em risco.
\end{abstract}

Palavras-chave: Doenças tropicais negligenciadas. Distribuição espacial. Ambiente. Saúde.

$\begin{array}{lllll}\text { Caminhos de Geografia } \quad \text { Uberlândia } & \text { v. 21, n. } 73 \quad \text { Mar/2020 } & \text { p. 518-529 Página } 518\end{array}$




\title{
SPACE DISTRIBUTION OF TROPICAL DISEASES NEGLECTED IN THE WEST REGION OF THE STATE OF SÃO PAULO
}

\begin{abstract}
This article aims to characterize the incidence of neglected tropical diseases in the western region of São Paulo between 2007 and 2017. It is a descriptive ecological study with a spatio-temporal approach, using the SINAN system as a data source, the Brazilian Institute of Geography and Statistics (IBGE). Five diseases were listed: Dengue, Leishmaniasis, Tuberculosis, Leprosy and Schistosomiasis in the 45 municipalities of the State of São Paulo that make up the Regional Health Assistance Network (RRAS), number 11, RRAS11. The incidence rate of diseases listed in each of the 45 municipalities of the RRAS was calculated by the ratio of the number of cases of the disease and the number of inhabitants in the municipality in 2010. Through the incidence rates it was possible to observe a heterogeneous distribution of these diseases in the 45 municipalities under study, with a greater incidence in the whole region of dengue and tuberculosis. The comparison of the incidence rates between the different municipalities of the West Paulista allowed us to identify the populations at risk
\end{abstract}

Keywords: Neglected tropical diseases. Spatial distribution. Environment. Health.

\section{INTRODUÇÃO}

As doenças negligenciadas são transmissíveis, causadas por agentes infecciosos e parasitários. Afetam cerca de 1,5 bilhão de pessoas, com prevalência registrada em 149 países, fazendo com que a própria doença perpetue os ciclos de precaridade, desigualdade e exclusão social, redução da produtividade da população trabalhadora e na promoção do estigma social (MOLYNEUX et al., 2017; HOTEZ, 2007)

Os estudos de distribuição espacial de doenças é objeto da geografia a muito tempo. A preocupação em estudar as relações entre o homem e o meio resultou em diversos trabalhos voltados à análise da distribuição espacial das doenças no mundo. Para Santos (1996) o espaço é a resultante da relação entre a materialidade das coisas e a vida que as animam e transformam, sendo a necessidade da economia o fator que determina sua reorganização. Sua tendência é mudar com o processo histórico, visto que o espaço geográfico é também o espaço social entendido como um conjunto indissociável de sistemas de ações e objetos.

Braga et al. (2001) enfatiza a necessidade de rever o conceito de espaço enquanto categoria de análise no sentido de compreender o processo de saúde-doença na coletividade. Com a urbanização ocorrida em todo o mundo, o interesse pelo cenário natural das doenças diminuiu, uma vez que a natureza intocada pela ação humana tornou-se quase inexistente, e a teoria de Pavlovsky, que por três décadas orientou as investigações da distribuição de doenças no espaço, tornou-se obsoleta.

Santos (2002) apresenta em sua obra que a transformação espacial não ocorre da mesma forma em todos os lugares, considerando que o espaço é um "campo de forças cuja aceleração é desigual" (p. 127). Portanto, o espaço geográfico está em constante processo de construção e mudanças.

Para Leal et al. (1992) foi Milton Santos que passou a estudar a distribuição das doenças como resultado da organização social do espaço, onde as sociedades humanas produziram uma segunda natureza por meio das transformações ambientais oriundas do processo de trabalho. 
Desta forma, as transformações espaciais e ambientais levaram a espaços de circulação e difusão de doenças, criando condições constantes e necessárias para a incidência e propagação de inúmeras moléstias.

A classificação atual proposta pela Organização Mundial da Saúde (OMS) é composta por 17 doenças como prioritárias para a eliminação até 2020. Algumas dessas doenças estão sendo combatidas através da administração em massa de doações de fármacos e envolvimento das comunidades e mudanças de comportamento. Para outras ainda existem grandes desafios, pois tem seu impacto e sua carga mal compreendida e ferramentas de controle ineficazes ou ultrapassadas (WHO, 2017).

Pessoas em situação de pobreza e privação de necessidades básicas de vida estão entre as mais vulneráveis à ocorrência de doenças, sobretudo, aquelas que vivem em locais com condições inadequadas de saneamento e em contato com vetores ou animais domésticos infectados (WHO, 2017). Doenças associadas à pobreza, à proliferação em ambientes empobrecidos e regiões tropicais são designadas comumente de Doenças Tropicais Negligenciadas (DTN). Recentemente tem se discutido a respeito das falhas que interferem no controle global das DTN, dentre elas podemos destacar: as instabilidades políticas e a falta de programas de controle; as mudanças climáticas e seus impactos nas populações de vetores; falha no acesso a serviços de saúde e a falta de medicamentos (HOTEZ, 2017).

O termo doenças negligenciadas se deve muito à dificuldade de se encontrar investimentos em pesquisas e desenvolvimento, pela baixa atenção no âmbito das políticas e dos serviços de saúde das agências nacionais e internacionais (CAMARGO, 2008).

Várias condições podem predispor o aparecimento e aumento de diversas doenças infectocontagiosas como níveis de precipitação, temperatura e umidade relativa do ar. Ademais, as mudanças climáticas, migrações, urbanizações e depósitos inadequados de lixos são outros aspectos relacionados pela alteração na distribuição de diversas doenças (SHORT et al., 2017; BOOTH, 2018).

As DTNs podem ser influenciadas pelas características particulares das áreas geográficas e pelo nível de agregação espacial, ou seja, a escala, onde acontece a materialização dos fenômenos. Desta forma, estudos com foco em análises espaciais para diversos tipos de doenças tem se destacado, uma vez que a população em risco é semelhante, numa determinada área ou região (BOOCKER, 2007).

O Brasil possui elevado número dessas doenças, figurando entre os países com o maior número de casos de leishmanioses e dengue do mundo todo. A distribuição das DNTs por estado no Brasil também mostra um dado importante, os estados com menor índice de desenvolvimento humano do país concentram as maiores taxas (LINDOSO; LINDOSO, 2009; HOTEZ; FUJIWARA, 2014).

Entretanto, até o presente momento, não se conhece a distribuição espacial conjunta das doenças tropicais negligenciadas na região Oeste do Estado de São Paulo, de modo que seja possível apontar as doenças que mais acometem os municípios dessa região, bem como se há a co-presença entre municípios pertencentes a esta região geográfica. Nesse sentido, este trabalho teve por objetivo caracterizar a taxa de incidência e a co-presença de doenças tropicais negligenciadas na região do Oeste do Estado de São Paulo entre os anos de 2007 e 2017.

\section{MATERIAIS E MÉTODOS}

Foi realizado um estudo ecológico descritivo, com abordagem espacial, na região do Oeste Paulista - SP, em específico nos 45 municípios que compõem a Rede Regional de Assistência à Saúde (RRAS) de número 11, a RRAS-11 (Figura 1). A fonte de dados para Dengue, Leishmanioses, Tuberculose, Hanseníase e Esquistossomose foram os casos notificados no Sistema de Informação de Agravos de

$\begin{array}{lllll}\text { Caminhos de Geografia Uberlândia } & \text { v. 21, n. 73 } & \text { Mar/2020 } & \text { p. 518-529 Página } 520\end{array}$


Antonio Chigogoro Titosse

Elivelton da Silva Fonseca

Marcus Vinicius Pimenta Rodrigues

Ana Paula Marques Ramos

Rogério Giuffrida

Distribuição espacial das doenças tropicais

negligenciadas na região oeste do estado de São Paulo

Alba Regina Azevedo Arana

Notificação - SINAN dos anos de 2007 a 2017, e os dados referente ao número de habitantes por município vieram do Instituto Brasileiro de Geografia e Estatística (IBGE), censo de 2010. Os dados das DNTs e das populações de cada município foram organizados foram organizados em tabelas, as quais, posteriormente, serviram para a construção de mapas temáticos em ambiente de Sistema de Informação Geográfica (SIG), aplicando o quintil como método de classificação para a geração de mapas temáticos.

Foram calculadas as taxas de incidência das doenças selecionadas para cada um dos 45 municípios que compõe a RRAS-11. A taxa de incidência foi calculada entre a razão dos números de casos de cada uma das doenças e o número de habitantes no município em 2010, o último censo disponível, multiplicadas por 1.000. Os mapas de taxa de incidência das DNTs para o período de 2007 a 2017 foram construídos no SIG ArcGIS 10.3. A figura 1 ilustra a distribuição da população nos municípios de estudo.

Figura 1 - Distribuição da população na área geográfica de interesse, a a RRAS 11, de Presidente Prudente.

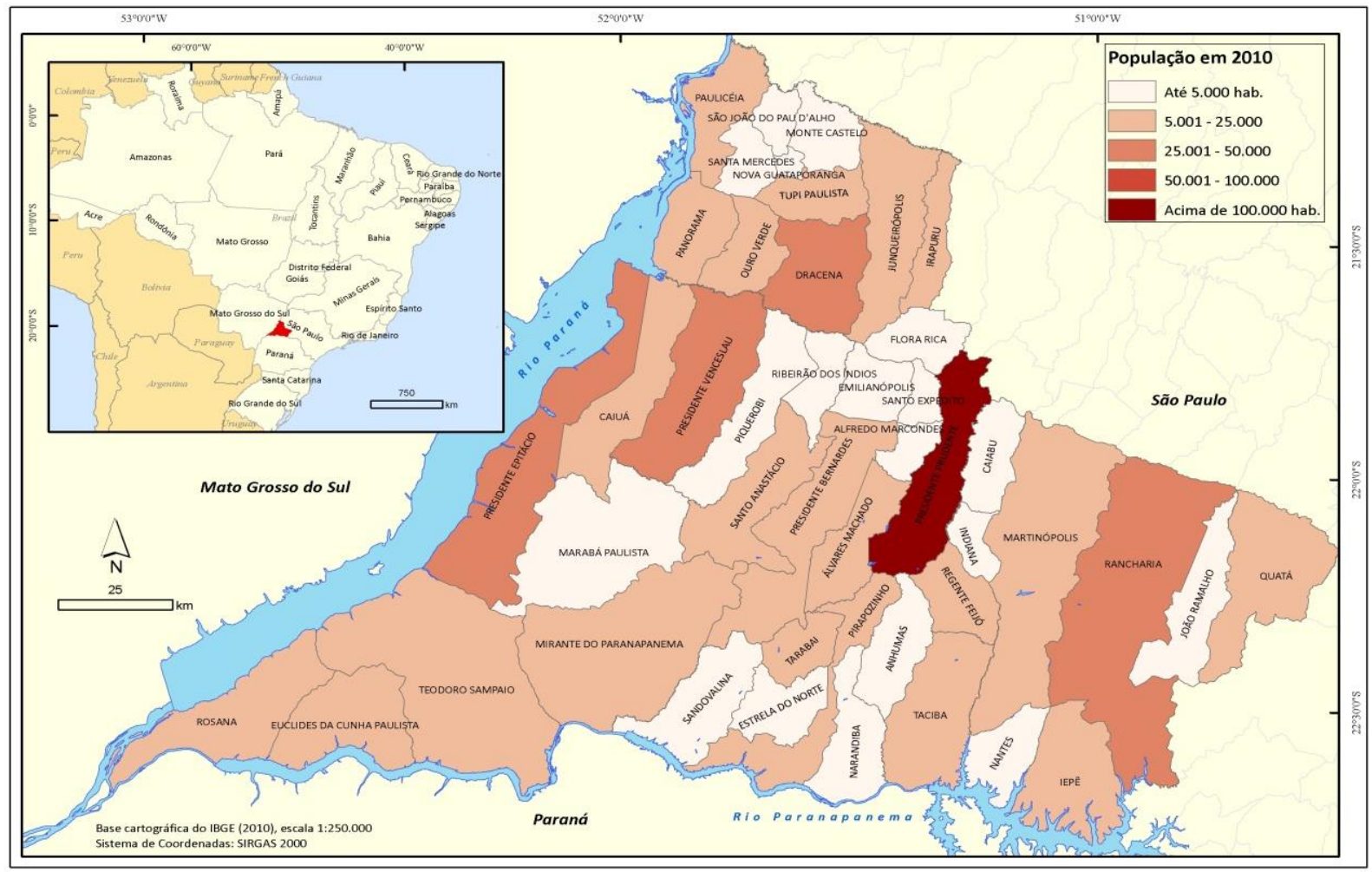

Fonte - os autores (2018).

\section{RESULTADOS E DISCUSSÃO}

A distribuição e a endemicidade das doenças tropicais e negligenciadas está comumente ligada a fatores que vão desde os aspectos geográficos, socioeconômicos, ambientais, mudanças climáticas, migração até a crescente urbanização das populações (BOOTH, 2018; HOTEZ; FUJIWARA, 2014).

A Tabela 1 apresenta a estimativa da população em risco no mundo, o número de novos casos por ano no país, dessas Doenças Tropicais Negligenciadas (DTNs). As DNTs não só afetam negativamente a saúde humana, mas também tem o potencial de afetar a economia local e mundial através da debilitação da saúde, impedindo a produtividade e o progresso das comunidades (SHORT et al., 2017). 
Antonio Chigogoro Titosse

Elivelton da Silva Fonseca

Marcus Vinicius Pimenta Rodrigues

Ana Paula Marques Ramos

Rogério Giuffrida

Distribuição espacial das doenças tropicais

negligenciadas na região oeste do estado de São Paulo

Alba Regina Azevedo Arana

Tabela 1 - Estimativas das populações em risco no mundo e no Brasil em contrair Doenças Tropicias Negligenciadas.

\begin{tabular}{|c|c|c|c|c|c|}
\hline Doença & $\begin{array}{l}\text { Agente } \\
\text { Etiológico }\end{array}$ & Vetor & $\begin{array}{l}\text { População } \\
\text { em risco no } \\
\text { mundo }\end{array}$ & $\begin{array}{c}\text { Novos } \\
\text { casos/ano } \\
\text { no Brasil }\end{array}$ & $\begin{array}{l}\text { Fonte de } \\
\text { informação }\end{array}$ \\
\hline $\begin{array}{c}\text { Dengue e outras } \\
\text { doenças } \\
\text { relacionadas à } \\
\text { arbovírus } \\
\text { (Chikungunya } \\
\text { vírus e Zika vírus) }\end{array}$ & $\begin{array}{l}\text { Flavivírus e } \\
\text { Togavírus }\end{array}$ & $\begin{array}{c}\text { Mosquito } \\
\text { (Aedes aegypti e } \\
\text { Aedes albopictus) }\end{array}$ & 3,9 Bilhões & 765.000 & $\begin{array}{c}\text { OMS, 2017; } \\
\text { Fiocruz, } 2012\end{array}$ \\
\hline Chagas & $\begin{array}{c}\text { Protozoário } \\
\text { Trypanossoma } \\
\text { cruzi }\end{array}$ & $\begin{array}{l}\text { Barbeiro } \\
\text { (Triatoma } \\
\text { infestans) }\end{array}$ & 75 Milhões & 200 & $\begin{array}{c}\text { OMS, 2017; } \\
\text { Brasília, } 2018\end{array}$ \\
\hline $\begin{array}{l}\text { Leishmaniose } \\
\text { Visceral }\end{array}$ & \multirow[t]{2}{*}{$\begin{array}{c}\text { Protozoários do } \\
\text { género } \\
\text { Leishmania }\end{array}$} & \multirow[t]{2}{*}{$\begin{array}{l}\text { Mosquito } \\
\text { flebotomíneos, } \\
\text { género Lutzomya } \\
\text { e Phlebotomus }\end{array}$} & \multirow[t]{2}{*}{350 Milhões } & 3.500 & \multirow[t]{2}{*}{$\begin{array}{c}\text { OMS, 17; } \\
\text { Fiocruz, 2012; } \\
\text { PAHO/WHO, } \\
17\end{array}$} \\
\hline $\begin{array}{c}\text { Leishmaniose } \\
\text { Tegumentar }\end{array}$ & & & & 28.000 & \\
\hline Hanseníase & $\begin{array}{c}\text { Bactéria } \\
\text { Mycobacterium } \\
\text { leprae }\end{array}$ & $\begin{array}{l}\text { Infectocontagiosa } \\
\text { "inalação de } \\
\text { goticulas de } \\
\text { saliva" } \\
\end{array}$ & & 34.000 & $\begin{array}{c}\text { OMS, 17; } \\
\text { Fiocruz, 2012 } \\
\text { PAHO/WHO, } \\
17 \\
\end{array}$ \\
\hline Malária & $\begin{array}{c}\text { Protozoário } \\
\text { Plasmodium } \\
\text { falciparum, } P \text {. } \\
\text { vivax }, P . \\
\text { malariae e } P \text {. } \\
\text { ovale }\end{array}$ & $\begin{array}{l}\text { Mosquito fêmea } \\
\text { (Anopheles) }\end{array}$ & 3,2 Bilhões & 300.000 & $\begin{array}{r}\text { OMS, 2015; } \\
\text { Fiocruz, } 2012\end{array}$ \\
\hline Esquistossomose & $\begin{array}{l}\text { Schistosoma } \\
\text { mansoni }\end{array}$ & $\begin{array}{c}\text { Caramujos de } \\
\text { água doce do } \\
\text { género } \\
\text { Biomphalaria } \\
\text { spp. E outros }\end{array}$ & 218.2 Milhões & & $\begin{array}{c}\text { OMS, 17; } \\
\text { PAHO/WHO, } \\
17\end{array}$ \\
\hline Tuberculose & $\begin{array}{c}\text { Bacteria } \\
\text { Mycobacterium } \\
\text { tuberculosis }\end{array}$ & $\begin{array}{l}\text { Infectocontagiosa } \\
\text { "inalação de } \\
\text { gotículas de } \\
\text { saliva" } \\
\end{array}$ & $\begin{array}{c}1 / 3 \mathrm{da} \\
\text { população } \\
\text { mundial }\end{array}$ & 69.000 & $\begin{array}{r}\text { OMS, 2017; } \\
\text { Fiocruz, } 2012\end{array}$ \\
\hline
\end{tabular}

Fonte - Adaptado da FIOCRUZ (2012)

A tabela 1 apresenta estimativas populacionais alarmantes relacionadas às Doenças Tropicais Negligenciadas. Pode-se observar que os números do Brasil em relação a dengue e as outras arboviroses (Chikungunya e Zika) são bastantes altos, chegando a 765.000 casos em 2012. Dados disponibilizados pelo Ministério da Saúde em 2018 aponta uma redução para 269.000 casos, contudo ainda é uma doença relevante com 3,9 bilhões de pessoas em risco. A Málaria também preocupa com 3,2 bilhões de pessoas em situação de risco, e no Brasil já estavam notificados 300 mil casos. Importante também destacar a Tuberculose, com cerca de $1 / 3$ da população mundial em risco e mais de 69 mil casos notificados no Brasil. A Leishmaniose preocupa o mundo com uma população de risco chegando a 350 milhões, no Brasil a Leishmaniose viseral chega a 3.500 casos e a Leishmaniose Tegumentar a 28 mil casos notificados.

Na região da Rede Regional de Assistência em Saúde - 11 (RRAS-11) do Estado de São Paulo, constatou-se que há cinco Doenças Tropicais Negligenciadas notificadas, de acordo com o levantamento no banco de dados do Sistema Nacional de Agravos e Notificação (SINAN) entre 2007 e 2017 (BRASIL,

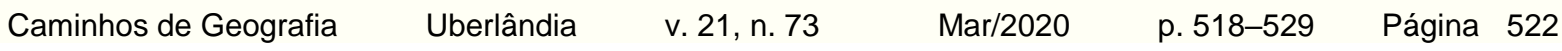


Antonio Chigogoro Titosse

Elivelton da Silva Fonseca

Marcus Vinicius Pimenta Rodrigues

Ana Paula Marques Ramos

Rogério Giuffrida

Distribuição espacial das doenças tropicais
negligenciadas na região oeste do estado de São Paulo

Alba Regina Azevedo Arana

2017). Dentre as DNTs notificadas no período estão: Dengue, Tuberculose, Leishmaniose, Hanseníase e Esquistossomose.

A figura 2 apresenta o mapa com a distribuição espacial com as taxas de incidência dessas DNTs notificadas na região da Rede Regional de Assistência em Saúde - 11(RRAS-11).

Figura 2 - Cidades que compõe a Rede Regional de Assistência em Saúde - 11(RRAS-11) e distribuição espacial das Doenças Tropicais Negligenciadas notificadas entre 2007 a 2017.

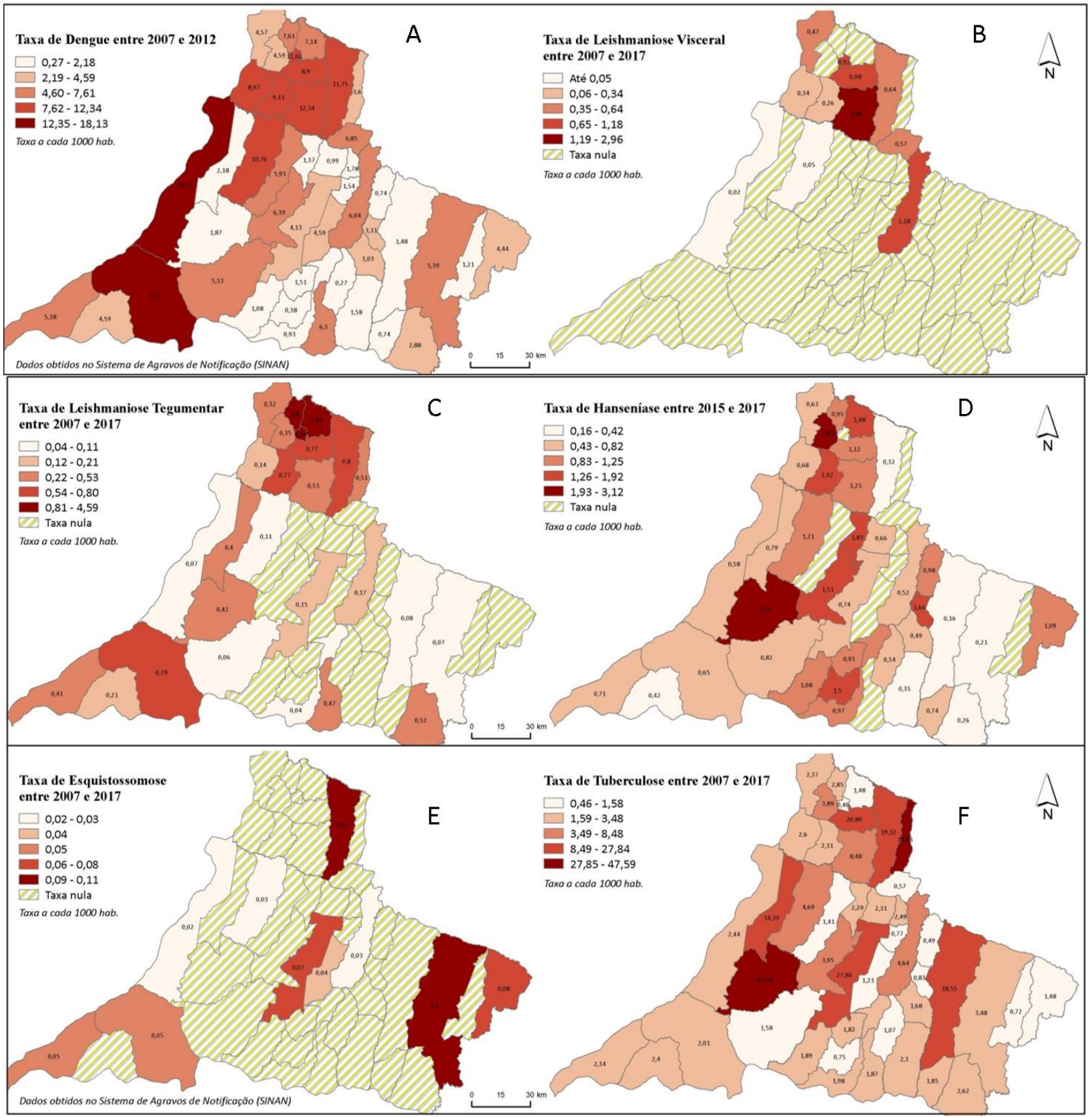

Fonte - Instituto Brasileiro de Geografia e estatística - IBGE (2010) e Sistema de Informação de Agravos de Notificação (2017).

A Dengue é a principal doença viral transmitida por mosquitos. Nas últimas décadas, a sua incidência tem aumentado de forma importante, afetando diferentes regiões no Brasil e no mundo, com especial atenção

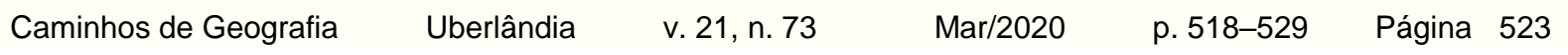


para Ásia e da América Latina (WHO, 2017). A Dengue é caracterizada por ser uma doença viral aguda, infecciosa, não contagiosa e sistêmica, são conhecidos atualmente quatro sorotipos, antigenicamente distintos: DEN-1, DEN-2, DEN-3 e DEN-4 (ARAÚJO-JORGE et al., 2017; SOO et al., 2016). A constante ocorrência de epidemias de Dengue no Brasil, aponta que o controle contínuo dessa doença, assim como as políticas de vigilância ainda precisam ser revistas. Evitar o reestabelecimento de focos de multiplicação desses mosquitos é uma condição prioritária, pois o clima predominantemente tropical do Brasil, torna o país suscetível a co-circulação de diferentes arboviroses. (FARES et al., 2015).

A Figura 2 indica no mapa A que todos 45 municípios da área analisada notificaram casos dessa doença e que sua distribuição espacial é heterogênea. Os municípios com maior incidência no período foram os municípios de Presidente Epitácio e Teodoro Sampaio, essas altas taxas podem estar relacionadas a proximidade do Parque Estadual "Morro de Diabo" e o turismo fluvial e rural que esse parque promove.

Um estudo ecológico, realizado por Carvalho et al. (2018), que analisaram a distribuição espacial da dengue, no estado de Rio de Janeiro no período de 2001 a 2012 observaram altas incidência para o período em estudo em municípios localizados nas regiões baixas litorâneas e norte fluminense. Esses dados coincidem com as altas incidências descritas nos municípios analisados no presente estudo.

Outro estudo sobre o perfil dos casos de Dengue desenvolvido por Ribeiro e colaboradores (2007) no município de São Sebastião-SP observaram que a área com maior frequência de casos apresentava como características a elevada densidade populacional, intenso fluxo turístico e comercial, além de serem áreas contíguas. O presente estudo apresenta dados similares, pois municípios com as maiores incidências dentre os analisados na RRAS - 11, como o município de Presidente Epitácio, por exemplo, é caracterizada como um município com importante atividade turística além de apresentar uma das maiores densidades populacional dentre as cidades da RRAS-11. Da mesma forma que o município de Rancharia que além da mesma característica turística também apresentou incidência de 5,39 a cada 1.000 hab.

A Leishmaniose é uma doença relacionada à pobreza com duas formas clínicas principais: leishmaniose visceral e a cutânea. Estima-se que 0,7 a 1 milhão de novos casos de Leishmaniose por ano são relatados em quase 100 países endêmicos para a doença (BURZA et al., 2018).

Na região da RRAS-11, constatou-se que houveram notificações de Leishmaniose Visceral (LV), no período do estudo, em 11 municípios (Figura 2, Mapa B), e que a taxa de incidência não ultrapassou 3,0 a cada 1.000 habitantes. Os municípios com maiores notificações dessa doença foram Dracena, seguido de Presidente Prudente, Tupi Paulista e Nova Guataporanga. Em se tratando da Leishmaniose Tegumentar (LT) (Figura 2, Mapa C), verificou-se um maior número de municípios com notificação de LT em relação às notificações de LV, da mesma forma as taxas de incidência de LT também foram mais altas, variando entre 0,04 e 4,59 casos notificados para 1.000 habitantes.

Cardim et al. (2015) em um estudo realizado no Estado de São Paulo observaram a expansão da Leishmaniose Visceral, no sentido Oeste-Leste do Estado. Esta expansão pode ser associada à alta concentração de cães infectados na região, expansão urbana e adaptação do vetor aos ecótipos urbanos e seres humanos, aglomerados urbanos, fluxo migratório das pessoas, produtos e serviços por rodovias e ferrovias, presença de pequenas propriedades rurais com material orgânico abundante, lixões e a dispersão do vetor. Estes dados podem justificar a distribuição da LT e LV na região avaliada no presente estudo

Em pesquisa sobre Leishmaniose Visceral na microrregião de Dracena em alta paulista, SP, Brasil, constatou-se que essa doença, está se espalhando de forma rápida e preocupante por todo o Oeste do Estado de São Paulo (LAZ et al., 2009). Outro estudo desenvolvido na mesma região onde avaliaram a prevalência de Leishmaniose Canina (LC), bem como a dispersão espacial da doença na região, indicou o movimento da doença através dos municípios dessa região do Estado de São Paulo em direção à fronteira do estado com o Estado do Paraná (D'ANDREA et al., 2015).

A Tuberculose (TB) continua sendo um grave problema da saúde pública mundial, mesmo sendo antiga e curável, ainda é uma das 10 principais causas de morte entre as doenças infectocontagiosa. Diversos fatores extrínsecos têm dificultado o controle dessa enfermidade, dentre eles: aglomeração das populações urbanas, condições socioeconômicas desfavoráveis, migrações, aumento de casos de HIV e aumento de tuberculose multirresistente (TBMDR). Ademais, fatores intrínsecos, como o estado

$\begin{array}{llllll}\text { Caminhos de Geografia } \quad \text { Uberlândia } & \text { v. 21, n. 73 } & \text { Mar/2020 } & \text { p. 518-529 } & \text { Página } 524\end{array}$


Antonio Chigogoro Titosse

Elivelton da Silva Fonseca

Marcus Vinicius Pimenta Rodrigues

Ana Paula Marques Ramos

Rogério Giuffrida

Distribuição espacial das doenças tropicais

negligenciadas na região oeste do estado de São Paulo

Alba Regina Azevedo Arana

imunológico do indivíduo, ambiente que está inserido e a duração da exposição ao bacilo, também contribuem para a dificuldade de controle da doença (DING et al., 2017; TEKLU et al., 2017).

A região avaliada no presente estudo apresentou uma heterogeneidade na distribuição espacial da taxa de incidênica de Tuberculoise durante o período analisado. As maiores taxas foram encontradas em sete municípios, dados similares aos apontados por de Bortoluci e colaboradores (2016) que avaliaram a distribuição espacial de Tuberculose na mesma região. Segundo esses autores, quatro dos sete municípios com as maiores taxas de TB possuem unidades prisionais superlotadas. A incidência de TB nas prisões brasileiras é superior ao da população em geral, mostrando maior risco de infecção por fator de aglomeração (VALÊNCIA et al., 2016).

Outro estudo sobre a distribuição espacial de Tuberculose desenvolvido em um município da região Centro-Oeste Paulista entre os anos de 2008 a 2013, aponta para a existência de aglomerados de casos em áreas alta vulnerabilidade social urbana, supostamente de riscos para a transmissibilidade da doença e/ou infecção. (FUSCO et al., 2017). Esse perfil pode justificar os dados apresentados no presente estudo, onde as mais altas incidências para Tuberculose foram observadas em municípios com alta vulnerabilidade social urbana.

A Hanseníase, tal como a Tuberculose, é uma enfermidade antiga, curável e de grande importância para a saúde pública, caracterizada como uma infecção granulomatosa crônica, com grande potencial de incapacidade, altamente contagiosa e de baixa mortalidade. A dinâmica precisa da transmissão da doença ainda não é claramente entendida, embora a transmissão pelo contato direto de pessoas tem sido apontada como uma das principais formas de transmissão. Tanto o bacilo vivo como o DNA do Mycobacterium leprae podem ser detectados no solo e na água de áreas endémicas, indicando um possível papel na transmissão dessa doença (BOOTH; CLEMENTS, 2018; MOHANTY et al., 2015).

A análise espacial da incidência de Hanseníase na região da RRAS-11 (Figura 2, mapa D) mostrou heterogeneidade entre os municípios, com uma variação entre 0,16 e 3,12, a cada 1.000 habitantes para os municípios de Martinópolis e Marabá Paulista respectivamente. Oito municípios (22,9\%) não apresentaram notificações de Hanseníase no período estudado.

Estudos têm apontado à associação da Hanseníase com as vulnerabilidades sociais da população com altas taxas de incidência de Hanseníase nos municípios do Brasil, destacando elevada taxa de analfabetismo, aglomerados populacionais, saneamento deficiente em maioria dos domicílios, crescente urbanização, desigualdade de renda e maior número de agregados familiares por domicílio (FREITAS et al., 2017).Outro estudo realizado na Amazônia relatou a associação entre o desmatamento e as elevadas taxas de incidências de Hanseníase, apontando as precariedades das condições dos municípios (SILVA et al., 2010). Esses estudos podem corroborar com a presença de casos em algumas cidades da RRAS11 que sofreram importante desmatamento para a implementação de culturas, assim como as condições sociais que a região apresenta, como baixa escolaridade, IDH baixo, dentre outros fatores.

A Esquistossomose é uma doença de caráter endêmico em áreas tropicais e subtropicais, está associada a fatores socioeconômicos, como pobreza, baixo desenvolvimento, saneamento; fatores ambientais, e antropogênicos, como urbanização e redirecionamento da água disponível para sistemas de irrigação e construção de barragens Estimativas apontam que 218,2 milhões de pessoas precisam de tratamento, sendo que as crianças com idade escolar representam mais da metade $(54,4 \%)$ desses casos. A carga global da doença é estimada em 3.514.145 DALYs (Disability-Adjusted Life Years). A região africana foi a mais afetada com cerca de, 92\% de pessoas necessitando de tratamento preventivo (WHO, 2018).

No Brasil, a esquistossomose é uma doença endêmica de grande importância na saúde pública, presente em 19 estados; com endemicidade em oito estados, principalmente as regiões Nordeste e Sudeste. Essa doença é de baixa letalidade, sendo as principais causas de óbitos relacionadas às formas clínicas mais graves (LINDOSO; LINDOSO, 2009; SANTOS et al., 2015).

No presente estudo a distribuição das taxas de incidência da Esquistossomose na região da RRAS-11, durante o período analisado apresentou taxas baixas variando entre 0,02 a 0,11 por 1.000 habitantes (Figura 2, mapa E). Dois municípios se destacaram com as maiores taxas, dentre os 35 municípios analisados, Rancharia e Junqueirópolis. A maioria dos municípios da RRAS-11 não apresentaram notificações dessa doença no período de análise.

Um estudo desenvolvido na busca por aspectos particulares do comportamento epidemiológico da Esquistossomose no Estado de São Paulo, não encontrou a transmissão autóctone na região. Isto reforça

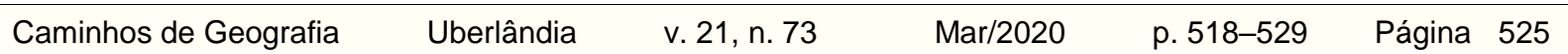


Antonio Chigogoro Titosse

Elivelton da Silva Fonseca

Marcus Vinicius Pimenta Rodrigues

Ana Paula Marques Ramos

Rogério Giuffrida

Distribuição espacial das doenças tropicais

negligenciadas na região oeste do estado de São Paulo

Alba Regina Azevedo Arana

o fato dos casos, hoje existentes, possivelmente, estarem associados ao contingente migratório ou de trabalho nas companhias de canavial, aglomerados populacionais, forte urbanização da região, saneamento deficitário e alta atividade agropecuária da região (CHIEFFI; WALDMAN, 2007). Esses fatores podem justificar a notificação dessa doença na região da RRAS-11, uma vez que essa região apresenta uma importante área de trabalho canavieiro e de outras atividades agropecuárias.

\section{CONSIDERAÇÕES FINAIS}

A comparação das taxas de incidência entre os diferentes municípios do oeste paulista nos permitiu identificar as populações em risco, criando um perfil das DTNs para a área de estudo. Também, através da identificação das variáveis que interferem em cada tipo de DN, pode-se identificar o impacto dessas doenças na região do oeste paulista. Outra importante constatação foi a sobreposição de duas ou mais DNs nos municípios investigados, o que caracteriza a co-presença dessas doenças permitindo apontar quais os municípios mais carentes de políticas públicas.

A pesquisa permitiu constatar que a dengue teve maior incidência nos municípios de Presidente Epitácio e Teodoro Sampaio. As notificações de Leishmaniose Visceral (LV) foram em Dracena, seguido de Presidente Prudente, Tupi Paulista e Nova Guataporanga, contudo constatou-se que essa doença, está se espalhando de forma rápida por todo o Oeste do Estado de São Paulo. Já a Tuberculose aparece em um município da região Centro-Oeste Paulista apontando para áreas alta vulnerabilidade social urbana. A ocorrência de Hanseníase mostrou-se bastante heterogênea nos municípios estudados, somente Martinópolis e Marabá Paulista apresentaram notificações da doença. A Esquistossomose também apresentou baixas taxas, somente Rancharia e Junqueirópolis apresentaram notificações dessa doença no período de análise.

A distribuição espacial foi relevante, pois apontou os locais que necessitam de maior aporte de intervenções, assim como de políticas sociais e de saúde pública direcionada ao combate desses agravos. Recomenda-se que os gestores e as equipes multiprofissionais de saúde possam, efetivamente, atuar no treinamento e informações para os profissionais de saúde e na prevenção, diagnóstico e tratamento da população em razão do aumento da incidência das DTNs na região.

\section{AGRADECIMENTOS}

Agradecemos a Coordenação de Bolsas de Aperfeiçoamento de Pessoal de Nível Superior (CAPES) pela bolsa concedida, sob o número 1706272/2017.

\section{REFERÊNCIAS}

ALENE K.A., VINEY K., MCBRYDE E.S., CLEMENTS A.C.A. Spatial patterns of multidrug resistant tuberculosis and relationships to socioeconomic, demographic and household factors in northwest Ethiopia. PLoS One. 2017. https://doi.org/10.1371/journal.pone.0171800

ARAÚJO J.T.C., SILVA-PIRES F.D.E.S., MELLO M.L.B.C., BONATTO M.P., TRAJANO V.D.S. As doenças negligenciadas e os determinantes sociais da saúde no contexto da investigação em ensino. Educ Ciência e Cult. 2017;22(1):51. https://doi.org/10.1371/journal.pone.0171800

BOOTH M., CLEMENTS A. Neglected Tropical Disease Control - The Case for Adaptive, Location-specific Solutions. Trends Parasitol. 2018. Apr;34(4):272-282. doi: 10.1016/j.pt.2018.02.001. Epub 2018 Feb 27. https://doi.org/10.1016/i.pt.2018.02.001

Climate Change and the Neglected Tropical Diseases [Internet]. 1st ed. Vol. 100, Advances in Parasitology. Elsevier Ltd.; 2018. 39-126 p. https://doi.org/10.1016/bs.apar.2018.02.001

BORTOLUCI A.B., RODRIGUES M.V.P.,CARRASCO R.A., TOMIAZZI J.S., RAMOS A.P.M. Avaliação Da Distribuição Espacial Da Tuberculose Na Região Do Pontal Do Paranapanema. Colloq Vitae. 2017;8(Especial):14-22. https://doi.org/10.5747/cv.2016.v08.nesp.000259

BRAGA C, XIMENES R.A.A., ALBUQUERQUE M.F.P.M., SOUZA W.V., MIRANDA J., BRAYNER F., ALVES L., SILVA L., DOURADO I. Avaliação de indicador sócio-ambiental utilizado no rastreamento de

$\begin{array}{lllll}\text { Caminhos de Geografia } \quad \text { Uberlândia } & \text { v. 21, n. 73 } & \text { Mar/2020 } & \text { p. 518-529 Página } 526\end{array}$


áreas de transmissão de filariose linfática em espaços urbanos. Cad Saude Publica 2001. 17(5):12111218. https://doi.org/10.1590/S0102-311X2001000500021

BRASIL. Ministério da Saúde. Secretaria de Vigilância em Saúde. Departamento de Vigilância Epidemiológica. Sistema de Informação de Agravos de Notificação - Sinan: normas e rotinas / Ministério da Saúde, Secretaria de Vigilância em Saúde, Departamento de Vigilância Epidemiológica. - 2. ed. Brasília : Editora do Ministério da Saúde, 2007

Ministério da Saúde. Secretaria de Vigilância em Saúde. Departamento de Vigilância Epidemiológica. Sistema de Informação de Agravos de Notificação , 2017

. Ministério da Saúde. Tegumentar americana. Ministério da Saúde. Manual de vigilância. 2013.

BROOKER S, UTZINGER J. Integrated disease mapping in a polyparasitic world. Geospat Health. 2007;1(2):141-6.

https://doi.org/10.4081/gh.2007.262

http://portalsinan.saude.gov.br/dados-epidemiologicos-sinan.

BURZA, S., CROFT, S. L., \& BOELAERT, M. (2018). Leishmaniasis. The Lancet. 2018. 392, issue 10151:951-970. https://doi.org/10.1016/S0140-6736(18)31204-2

CAMARGO, Erney Plessmann. Tropical diseases. Estud. $2008 . \quad 22(64): 95-110$. https://doi.org/10.1590/S0103-40142008000300007

CARDIM M.F.M., VIEIRA C.P., CHIARAVALLOTI-NETO F. Spatial and spatiotemporal occurrence of human visceral leishmaniasis in Adamantina, State of São Paulo, Brazil. Rev Soc Bras Med Trop. 2015.48(6):716-23. https://doi.org/10.1590/0037-8682-0213-2015

CARVALHO, C.O.; RABELLO, R.S.; THOMÉ S. Distribuição espacial da dengue no estado do Rio de Janeiro no período de 2001 a 2012. Hygeia. 2018.14(27):108-23.

CHIEFFI P.P., WALDMAN E.A. Aspectos particulares do comportamento epidemiológico da esquistossomose mansônica no Estado de São Paulo, Brasil. Cad Saude Publica. 2007.4(3):257-75. https://doi.org/10.1590/S0102-311X1988000300002

D'ANDREA L.A., FONSECA E.DA S., PRESTES-CARNEIRO L.E., GUIMARÃES R.B., YAMASHITA R.C., SOARES, C.N.. The shadows of a ghost: a survey of canine leishmaniasis in Presidente Prudente and its spatial dispersion in the western region of São Paulo state, an emerging focus of visceral leishmaniasis in Brazil. BMC Vet Res. 2015. 11:273. Published 2015 Oct 26. https://doi.org/10.1186/s12917-015-0583-6

DING P., LI X, J.I.A.Z,. LU Z. Multidrug-resistant tuberculosis (MDR-TB) disease burden in China: A systematic review and spatio-temporal analysis. BMC Infect Dis. 2017.17(1):1-29. https://doi.org/10.1186/s12879-016-2151-5

ERNEY P.C.. Doenças tropicais. Estud Avançados. 2008. 22(64):95-110. https://doi.org/10.1590/S0103-40142008000300007

FARES R.C.G., SOUZA K.P.R., AÑEZ G., RIOS M. Epidemiological Scenario of Dengue in Brazil. Biomed Res Int. 2015. 2015:1-13. https://doi.org/10.1155/2015/321873

FREITAS L.R.S. de, DUARTE E.C., GARCIA L.P. Análise da situação epidemiológica da hanseníase em uma área endêmica no Brasil: distribuição espacial dos períodos 2001 - 2003 e 2010 - 2012. Rev Bras Epidemiol. 2017. 20(4):702-13. https://doi.org/10.1590/1980-5497201700040012

FUSCO A.P.B., ARCÊNCIO R.A., YAMAMURA M., PALHA P.F., REIS A.A. dos, ALECRIM T.F. de A. Spatial distribution of tuberculosis in a municipality in the interior of São Paulo, 2008-2013. Rev. LatinoAm. Enfermagem. $\quad$ v. 25, e2888. $2017 . \quad$ Available from <http://www.scielo.br/scielo.php?script=sci_arttext\&pid=S0104-11692017000100340\&lng=en\&nrm=iso>. access on 15 Oct. 2019. Epub June 05, 2017. https://doi.org/10.1590/1518-8345.1064.2888

HOTEZ P. A New Voice for the Poor. Plos Negl Trop Dis. 2007;1(1):e77. https://doi.org/10.1371/journal.pntd.0000077

HOTEZ P.J., FUJIWARA R.T. Brazil's neglected tropical diseases: an overview and a report card. Microbes Infect. 2014.16(8):601-6. https://doi.org/10.1016/i.micinf.2014.07.006 
Antonio Chigogoro Titosse

Elivelton da Silva Fonseca

Marcus Vinicius Pimenta Rodrigues

Ana Paula Marques Ramos Rogério Giuffrida

Distribuição espacial das doenças tropicais

negligenciadas na região oeste do estado de São Paulo

Alba Regina Azevedo Arana

IBGE- Instituto Brasileiro de Geografia e estatística ,Censo Demográfico. 2010. https://www.ibge.gov.br/home/estatistica/populacao/censo2010/default.shtm

LASTÓRIA J.C.L., ABREU, M.A.M.M.. Hanseníase: diagnóstico e tratamento. Diagn Trat. 2012.17(4):5-8.

LAZ D., CAMARGO-NEVES V.L.F., SAMPAIO S., KRONKA S., SARTOR I. American Visceral LEishmaniasis: Disease control strategies in Dracena Macroregion in Alta Paulista, SP, Brasil. J Venom Anim Toxins Incl Trop Dis. 2009.15(2):305-24. https://doi.org/10.1590/S1678-91992009000200012

LEAL M., SABROZA P.C., RODRIGUES R., BUSS P. Saúde, ambiente e desenvolvimento. Rio de Janeiro: Abrasco; 1992.

LINDOSO J., LINDOSO A.. Neglected tropical diseases in Brazil. Rev Inst Med Trop. . 2009. 51(5):24753. Available from: http:/www.scielo.br/scielo.php?script=sci arttext\&pid=S00366652009000500003\&lng=en\&tlng=en. https://doi.org/10.1590/S0036-46652009000500003

MOHANTY P., MISBA L., DWIVEDI D., KATARA D., KUMAR D., BANSAL A. Viability of Mycobacterium leprae in the environment and its role in leprosy dissemination. Indian J Dermatology, Venereol Leprol. 2015. 82(1):23. https://doi.org/10.4103/0378-6323.168935

MOLYNEUX D.H., SAVIOLI L., Engels D. Neglected tropical diseases: progress towards addressing the chronic pandemic. Lancet [Internet]. 2017;389(10066):312-25. Available from: http://dx.doi.org/10.1016/S0140-6736(16)30171-4

OMS- Organização Mundial da Saúde, Relatório de Mundial de Saúde, 2017 https://apps.who.int/iris/bitstream/handle/10665/255336/9789241565486eng.pdf;jsessionid=CAB33AE637D1970DB74B498EB5B38ED4?sequence $=1$

RIBEIRO A.F., SERPA L.L.N., LEFÈVRE F., MARQUES G.R.A.M., LEFÈVRE A.M.C.. Representações sobre dengue, seu vetor e ações de controle por moradores do município de São Sebastião, litoral Norte do Estado de São Paulo, Brasil. Cad Saude Publica. 2007. 23(7):1696-706. https://doi.org/10.1590/S0102-311X2007000700022

SANTOS A.D.S, BEZERRA S., MACHADO D.K.C.G.. Análise do Grau de Implantação (Gl) do Programa de Controle da Esquistossomose Mansônica em um município endêmico do estado de Sergipe. Rev Iberoam Educ E Investig en Enfermería. 2015. 5(4):40-9.

SANTOS M. A natureza do espaço- técnica e tempo, razão e emoção. São Paulo: Hucitec; 1996.

Por uma geografia nova. São Paulo: EDUSP; 2002

SÃO PAULO. Manual de Vigilância e Controle da Leishmaniose Visceral Americana do Estado de São Paulo. Secr Estado da Saúde. 2006;161.

SAÚDE FN de, Fundação Nacional de Saúde. Manual de controle da Leishmaniose tegumentar americana. Ministério da Saúde. 2000;1-62.

SHORT E.E., CAMINADE C., THOMAS B.N.. Climate Change Contribution to the Emergence or ReEmergence of Parasitic Diseases. Infectious Diseases: Research and Treatment. 2017. 10: 1-7. https://doi.org/10.1177/1178633617732296

SILVA DRX, IGNOTTI E, SOUZA-SANTOS R, HACON S de S. Hanseníase, condições sociais e desmatamento na Amazônia brasileira. Rev Panam Salud Pública. 2010. 27(4):268-75. https://doi.org/10.1590/S1020-49892010000400005

SISAN- Sistema de Informação de Agravos de Notificação -.2017.http://portalsinan.saude.gov.br/sinan

SOO K.M., KHALID B., CHING S.M., CHEE H.Y.. Meta-analysis of dengue severity during infection by different dengue virus serotypes in primary and secondary infections. PLoS One. 2016.11(5):4-14. https://doi.org/10.1371/journal.pone.0154760

TEKLU A.M., NEGA A., MAMUYE A.T., SITOTAW Y., KASSA D., MESFIN G.. Factors Associated with Mortality of TB/HIV Co-infected Patients in Ethiopia. Ethiop J Health Sci. 2017.27(Suppl 1):29-38. https://doi.org/10.4314/ejhs.v27i1.4S 
Antonio Chigogoro Titosse

Elivelton da Silva Fonseca Marcus Vinicius Pimenta Rodrigues

Ana Paula Marques Ramos

UNICEF/UNDP/World Bank/WHO Special Programme for Research and Training in Tropical Diseases., World Health Organization. Research to support the elimination of visceral leishmaniasis: annual report 2008. TDR Bus line 10 [Internet]. 2009;27 p. Available from: http://whqlibdoc.who.int/hq/2009/TDR BL.10 eng.pdf

VALENÇA M.S., POSSUELO L.G., CEZAR-VAZ M.R., SILVA P.E.A.. Tuberculose em presídios brasileiros: uma revisão integrativa da literatura. Cien Saude Colet. 2016.21(7):2147-60. https://doi.org/10.1590/1413-81232015217.16172015

VIRGINIA C, ROLLEMBERG V, MARIA C, SANTOS B, MATOS M, LEMOS B. Esquist. e geoelmintos MG. 2011;44(1):91-6.

WORLD HEALTH ORGANIZATION. Global Health Estimates 2016: Disease burden by Cause, Age, Sex, by Country and by Region, 2000-2016. [Internet]. Geneva, 2018. Available from: https://www.who.int/healthinfo/global_burden_disease/estimates/en/index1.html__Fourth WHO report on neglected tropical diseases. World Health Organization. 2017. Fact Sheet March 2017.

Recebido em: 01/08/2019

Aceito para publicação em: 16/10/2019 